\title{
Prevalence and Clinical Management of Non-malarial Febrile Illnesses among Outpatients in the Era of Universal Malaria Testing in Malawi
}

\author{
Atupele Kapito-Tembo, ${ }^{1}$ Don Mathanga, ${ }^{1}$ Andrew Bauleni, ${ }^{1}$ Osward Nyirenda, ${ }^{2}$ Paul Pensulo, ${ }^{2}$ Doreen Ali, ${ }^{3}$ Clarissa Valim, ${ }^{4}$ \\ Terrie E. Taylor, ${ }^{2,5}$ and Miriam K. Laufer ${ }^{6 *}$ \\ ${ }^{1}$ Malaria Alert Center, University of Malawi College of Medicine, Blantyre, Malawi; ${ }^{2}$ Blantyre Malaria Project, University of Malawi College of \\ Medicine, Blantyre, Malawi; ${ }^{3}$ Malawi National Malaria Control Program, Lilongwe, Malawi; ${ }^{4}$ Department of Immunology and Infectious Diseases, \\ Harvard School of Public Health, Boston, Massachusetts; ${ }^{5}$ College of Osteopathic Medicine, Michigan State University, East Lansing, Michigan; \\ ${ }^{6}$ Center for Vaccine Development and Global Health, University of Maryland School of Medicine, Baltimore, Maryland
}

\begin{abstract}
Increasing access to rapid diagnostic tests for malaria (mRDTs) has raised awareness of the challenges healthcare workers face in managing non-malarial febrile illnesses (NMFIs). We examined NMFI prevalence, clinical diagnoses, and prescribing practices in outpatient clinics across different malaria transmission settings in Malawi. Standardized facility-based malaria surveillance was conducted at three facilities one of every 4 weeks over 2 years. Information on demographics, presenting symptoms, temperature, clinical diagnosis, and treatment were collected from outpatients presenting with malaria-like symptoms. Of the 25,486 patients with fever, $69 \%$ had NMFI. Non-malarial febrile illness prevalence was lower in 5- to 15-year-old patients (55\%) than in children $<5$ years (72\%) and adults $>15$ years of age (77\%). The most common clinical diagnoses among febrile patients with negative mRDTs in all age-groups and settings were respiratory infections (46\%), sepsis (29\%), gastroenteritis (13\%), musculoskeletal pain (9\%), and malaria (5\%). Antibiotic prescribing was high in all age-groups and settings. Trimethoprim-sulfamethoxazole (40\%) and amoxicillin (29\%) were the most commonly prescribed antibiotics and were used for nearly all clinical diagnoses. In these settings with minimal access to diagnostic tools, patients with fever and a negative mRDT received a limited number of clinical diagnoses. Many were likely to be inaccurate and were associated with the inappropriate use of the limited range of available antibiotics. Prescription and diagnostic practices for NMFls in the facilities require research and policy input. Resource-limited malaria-endemic countries urgently need more point-of-care diagnostic tools and evidence-based diagnosis and treatment algorithms to provide effective and cost-efficient care.
\end{abstract}

\section{INTRODUCTION}

The WHO guidelines recommend parasitological confirmation of all patients suspected of malaria before treatment. ${ }^{1}$ With widespread usage of malaria rapid diagnostic tests (mRDTs), there is increasing evidence that many febrile illnesses previously treated presumptively as malaria in endemic areas are of non-malarial origin. ${ }^{2,3}$ Health workers experience difficulties evaluating patients with fever when the mRDT is negative. ${ }^{4}$ They lack skills and resources such as point-of-care diagnostics and simple evidence-based management algorithms to facilitate accurate diagnosis and treatment of patients with non-malarial febrile illnesses (NMFls). ${ }^{4-8}$ In the absence of guidelines, health workers may inaccurately diagnose and treat patients with NMFIs which might result in poor patient outcomes and increased healthcare costs.

Although there has been a growing interest in studying NMFIs in malaria-endemic settings, previous studies have mainly focused on identifying the etiological agents. ${ }^{9-13}$ There is limited information on the distribution patterns of the most common routine clinical diagnoses and prescription practices by health workers in patients with NMFIs in malaria-endemic areas. $^{8,14}$ In addition, although there is a wealth of literature on the burden and clinical management of fevers in children younger than 5 years in sub-Saharan Africa from studies and reviews, only few studies have included older children and adults. $^{15-18}$ Recognition of NMFI burden, most common clinical diagnoses, and prescribing practices in patients with NMFls will help prioritize future diagnostics and treatment

\footnotetext{
*Address correspondence to Miriam K. Laufer, Center for Vaccine Development and Global Health, University of Maryland School of Medicine, 685 W. Baltimore St., HSF-1 Rm. 480, Baltimore, MD 21201. E-mail: mlaufer@som.umaryland.edu
}

algorithms. This evidence may provide information on healthcare planning, patient care, and evidence-driven allocation of resources such as drugs and diagnostics and help prioritize health conditions that need further investigation to better understand local epidemiology of common NMFIs and their best management and control approaches. Current data on the most frequent clinical diagnoses may also guide the process of identifying locally relevant point-of-care diagnostic tools to improve diagnosis accuracy.

We describe the prevalence, distribution patterns of routine clinical diagnoses, and prescribing practices in outpatients of all age-groups with NMFIs in Malawi using data collected during a facility-based malaria surveillance study conducted in three geographical settings. We also assessed clinical diagnostic distribution patterns with respect to season, location, malaria endemicity, age, and healthcare level.

\section{METHODS}

Study sites. The study was conducted from January 2012 to December 2013 at Ndirande Health Center in Blantyre city and at Thyolo and Chikhwawa rural district hospitals, all located in southern Malawi. All the three facilities provide free health services and have a subtropical climate, and rainy and dry seasons from November to April and May to October, respectively. ${ }^{19}$

Ndirande Health Center is located in a moderate stable malaria transmission area. ${ }^{20}$ The health center is located in Blantyre district which is situated in the Shire highland area with an elevation range of 780-1,612 $\mathrm{m}$ above sea level with an average annual temperature of $21^{\circ} \mathrm{C}$ and a rainfall of $1,127 \mathrm{~mm}^{19,21}$ In 2013, the health center had 104,867 outpatient visits (Ministry of Health, Health Management Information System data). 
Thyolo district hospital is located in a moderate stable malaria transmission area. ${ }^{20}$ The district is situated in the Shire highland area that has an elevation range of 780-1,612 m above sea level and has an average annual temperature of $22^{\circ} \mathrm{C}$ and a rainfall of $1,200 \mathrm{~mm} .{ }^{19,21}$ In 2013, the hospital had 141,666 outpatient visits (Ministry of Health, Health Management Information System data).

Chikhwawa district hospital is located in an intense stable malaria transmission area. ${ }^{20}$ The district is situated in the lowlying humid areas of the Shire River valley, approximately $70 \mathrm{~m}$ above sea level, and is divided throughout its length by the river which is prone to flooding in the rainy season. ${ }^{19}$ The average annual temperature and rainfall are $28^{\circ} \mathrm{C}$ and $770 \mathrm{~mm}$, respectively. ${ }^{19}$ In 2013 , the hospital had 76,372 outpatient visits (Ministry of Health, Health Management Information System data).

Study design. Facility-based malaria surveillance was conducted at the outpatient department 1 week of every 4 weeks in each of the three health facilities. Study staff obtained basic patient demographic information and measured axillary temperature of all patients seeking care in the waiting area. Study staff only worked in the patient waiting area before being seen by a clinician and at the patient exit point where data were collected from the health passport. All patients were evaluated by government facility clinicians who determined whether to order a malaria test. In all the facilities, malaria testing (either mRTD or blood smear) was the only diagnostic testing available for outpatients on a routine basis. Data were collected from all patients who had a malaria test performed. At the time of data entry, the written information from the patient's records was transcribed by the study staff from the health passport into electronic case report forms. Data collected included axillary temperature, basic demographics, history of fever, malaria test results, final diagnoses, prescribed treatment, and disposition. For this analysis, we included patients with a documented fever or who reported a history of fever that was recorded in the health passport, and who were also tested for malaria.

Ethical considerations. The study was reviewed and approved by the University of Malawi, College of Medicine Research and Ethics Committee, and the Institutional Review Boards of the Michigan State University and University of Maryland, Baltimore.

Laboratory tests. Two types of the histidine-rich protein-2 mRDT SD BIOLINE malaria Ag P.f (Standard Diagnostics, Inc., Hagai-dong, Giheung-gu, Yongin-si, Gyeonggi-do, South Korea) or Paracheck malaria Ag Pf (Orchid Biomedical Systems, Goa, India) were used in all the health facilities in accordance with national policy. A thick blood film was only prepared and read at the facility when mRDTs were not available.

Definitions. Fever was defined as a reported history of fever or an axillary temperature of $\geq 37.5^{\circ} \mathrm{C}$ measured at the time of presentation. Non-malarial febrile illness was defined as fever and a negative malaria test. Age categories were defined as young children (aged $<5$ years), school-aged children (aged 5-15 years), and adults (aged $>15$ years). Clinical diagnosis was transcribed from the patient's records as indicated by the attending government healthcare worker. Diagnoses were grouped as follows: 1) respiratory tract infections (RTIs) included diagnoses of acute respiratory, respiratory tract, upper respiratory tract, and lower respiratory tract infections (LRTIs); 2) upper respiratory tract infections (URTIs) included diagnoses of URTI, cold, common cold, flu, sore throat, and coryza, with exclusion of otitis media and tonsillitis; 3) LRTIs included diagnoses of pneumonia, LRTIs, and bronchitis; 4) soft tissue infection included diagnoses of cellulitis, abscess, impetigo, and soft tissue infection; 5) gastroenteritis (GE) included diagnoses of acute diarrhea, diarrhea, acute GE, and $\mathrm{GE}$, with exclusion of dysentery, shigellosis, and amebiasis; and 6) other diseases included diagnoses of cancer, congenital abnormalities, diabetes, epilepsy, hypertension, cardiovascular diseases, and renal failure 7) A malaria diagnosis was assigned if it was recorded as final diagnosis in the health passport by the government clinician on the clinic visit day. Treatment was as recorded in the patient's records by the attending government healthcare worker.

Statistical analysis. Data were analyzed using SAS version 9.4. Frequencies, percentages, and their $95 \%$ Cls were used to describe and analyze categorical data; means, 95\% Cls, median, and interquartile range (IQR) were used for continuous data, as appropriate. In bivariate analyses, The Pearson chi-square test or Fisher's exact test was used for categorical variables. Analysis of variance and Student's $t$-test were used to compare differences across groups, when reporting means. The Kruskal-Wallis test or Wilcoxon rank sum test was used to compare difference across groups when reporting medians. Although antipyretic and analgesic medications were analyzed, these medications were not included in the reports because they are not diagnosis-specific. Risk factors for antibiotic prescribing were evaluated through odds ratios and logistic regression models including malaria endemicity, age, axillary temperature, clinical diagnosis, and season. To perform model selection, we first screened all variables that were significant predictors of antibiotic prescribing using bivariate analysis to a significance level of 0.20 . Next, we evaluated combination of these screened variables and retained in the final model covariates with a $P$-value of 0.05 or less, or which caused a substantial change in the coefficients of other variables in the model. The purpose of the logistic multivariable model was to evaluate different predictors of antibiotic therapy and not a single exposure variable.

\section{RESULTS}

Study population. Of the total 51,446 outpatients enrolled with malaria-like symptoms, half of the participants $(25,486)$ had a measured fever or history of fever. Table 1 shows characteristics of the enrolled outpatients with fever and who were tested for malaria. Across all malaria transmission settings, a higher proportion of febrile outpatients tested for malaria were young children and females.

Prevalence of NMFI. Of the 25,486 patients with measured fever or history of fever and who were tested for malaria, $17,580(69 \%)$ met the definition of NMFI (Table 2). The proportion of NMFI was lowest in school-aged children (55\%) compared with both young children (72\%) and adults (77\%) in all settings. The proportion of NMFI was significantly higher in moderate transmission highlands of Ndirande and Thyolo than that in the intense transmission lowland of Chikhwawa during the dry season across all sites. Ndirande health center had a higher proportion of NMFI than rural district hospitals (81\% versus $64 \% ; P \leq 0.0001$ ).

Clinical diagnoses among NMFI. Among 17,580 patients with NMFI, 14,680 (84\%) had at least one clinical diagnosis. Of 
TABLE 1

Distribution of febrile outpatients tested for malaria with negative malaria tests in southern Malawi*

\begin{tabular}{|c|c|c|c|c|}
\hline Characteristic & Chikhwawat & Thyolot & Ndirande $\dagger$ & All sites \\
\hline Febrile outpatients tested for malaria & $(N=10,648)$ & $(N=7,957)$ & $(N=6,881)$ & $(N=25,486)$ \\
\hline $\begin{array}{l}\text { Female, } n(\%) \\
\text { Age category (years), } n \text { (\%)‡ }\end{array}$ & $5,763(54)$ & $4,266(54)$ & $3,649(53)$ & $13,678(54)$ \\
\hline$<5$ & $5,823(55)$ & $4,910(62)$ & 2,969 (43) & $13,694(54)$ \\
\hline $5-15$ & $2,796(26)$ & $1,415(18)$ & $1,787(26)$ & $5,998(24)$ \\
\hline$>15$ & $1,973(19)$ & $1,617(20)$ & $2,106(31)$ & $5,696(22)$ \\
\hline Median age, years (IQR) & $4.0(1.5-11.0)$ & $3.0(1.3-11.0)$ & $6.0(2.0-20.0)$ & $4.0(1.5-13.0)$ \\
\hline Dry season, $n(\%)$ & $5,427(51)$ & $3,374(42)$ & $3,474(50)$ & $12,275(48)$ \\
\hline
\end{tabular}

those with at least one diagnosis, 13,073 (89\%) had a single diagnosis, $1,570(11 \%)$ had two diagnoses, and $<1 \%$ had more than two diagnoses. The most common clinical diagnoses were RTIs (46\%), sepsis (29\%), GE (12\%), musculoskeletal pain (9\%), and malaria (5\%). Among the RTIs, 54\% were reported as upper RTI and $13 \%$ were lower RTI. Other diagnoses such as urinary tract infection, otitis media or tonsillitis, soft tissue infections, anemia, and noncommunicable diseases were uncommon with a prevalence $\leq 1 \%$ each.

The distribution pattern of common clinical diagnoses was similar during the rainy and dry seasons but showed some variation with age, malaria endemicity, and settings (Figure 1). Respiratory tract infections and sepsis were the most common diagnoses in all age-groups, study sites, and seasons. Musculoskeletal pain was frequently diagnosed in schoolaged children and adults, whereas GE was frequently diagnosed in young children compared with both school-aged children and adults. Malaria, despite a negative malaria test, was occasionally diagnosed among school-aged children compared with both young children and adults.

Medication prescription patterns. Antibiotics were the most commonly prescribed medications in all the three sites among outpatients with NMFI who received treatment, 13,581 of $17,322(78 \%)$. Antibiotic prescribing was high in all agegroups but decreased with increasing age, with a prevalence of $83 \%, 78 \%$, and $68 \%$ in the young children, school-aged children, and adults, respectively $(P \leq 0.0001)$.

Among outpatients with NMFI with a single diagnosis of URTI, LRTI, and sepsis, almost all were prescribed antibiotics, in almost all cases (95-99\%), and these proportions were similar in the three sites. Also, antibiotics were prescribed to patients with single diagnoses of malaria and musculoskeletal pain in $16 \%$ and $12 \%$ of cases, respectively. Cotrimoxazole $(40 \%)$ and amoxicillin (29\%) were the most commonly prescribed antibiotics. For patients with a single URTI diagnosis, $50 \%$ and $38 \%$ were prescribed cotrimoxazole and amoxicillin, respectively. These two drugs were prescribed for nearly all diagnoses including LRTI, sepsis, musculoskeletal pain, and malaria (Figure 2). Other antibiotics such as chloramphenicol, ciprofloxacin, and cephalosporins were rarely prescribed.

Predictors of antibiotic prescribing among NMFI patients. In multivariable analysis, age, malaria endemicity, and clinical diagnosis were statistically significantly associated with antibiotic prescribing practices (Table 3 ). When all these factors were simultaneously included in models, the odds of prescribing antibiotics was significantly higher in children younger than 5 years than those $>15$ years (odds ratio $[\mathrm{OR}]=1.37 ; 95 \% \mathrm{Cl}=$ 1.29-1.45). Compared with the urban Ndirande health center, the odds of prescribing antibiotics was significantly higher in rural Thyolo district hospital $(\mathrm{OR}=1.21 ; 95 \% \mathrm{Cl}=1.13-1.29)$

TABLE 2

Prevalence of NMFI among outpatients by characteristics and site

\begin{tabular}{|c|c|c|c|c|}
\hline & Chikhwawa* $^{*}$ & Thyolo* & Ndirande $^{*}$ & All sites \\
\hline & $N=10,648 n(\%$ with NMFI) & $N=7,957 n(\%$ with NMFI) & $N=6,881 n$ (\% with NMFI) & $N=25,486 n$ (\% with NMFI) \\
\hline Overall proportion with NMFI & $5,737(54)$ & 6,245 (78) & $5,598(81)$ & $17,580(69)$ \\
\hline \multicolumn{5}{|l|}{ Gendert } \\
\hline Female & $3,194(55)$ & $3,380(79)$ & 2,996 (82) & $9,570(70)$ \\
\hline Male & 2,537 (52) & $2,857(78)$ & $2,588(81)$ & $7,982(68)$ \\
\hline$P$-value & 0.0006 & 0.08 & 0.09 & \\
\hline \multicolumn{5}{|l|}{ Age category (years) $\dagger$} \\
\hline$<5$ & $3,120(54)$ & $4,149(85)$ & $2,552(86)$ & $9,821(72)$ \\
\hline $5-15$ & $1,176(42)$ & $830(59)$ & $1,315(74)$ & 3,321 (55) \\
\hline$>15$ & $1,409(71)$ & $1,255(78)$ & $1,709(81)$ & $4,373(77)$ \\
\hline$P$-value & $\leq 0.0001$ & $\leq 0.0001$ & $\leq 0.0001$ & \\
\hline \multicolumn{5}{|l|}{ Season† } \\
\hline Rainy & $2,720(52)$ & $3,299(72)$ & $2,630(77)$ & $8,649(65)$ \\
\hline Dry & $3,017(56)$ & $2,946(87)$ & $2,968(85)$ & $8,931(73)$ \\
\hline$P$-value & 0.0003 & $\leq 0.0001$ & $\leq 0.0001$ & \\
\hline
\end{tabular}

$\mathrm{NMFI}=$ non-malarial febrile illnesses.

* Malaria endemicity: Chikhwawa = rural lowland with intense stable transmission; Thyolo = rural highland with moderate stable transmission; Ndirande = urban highland with moderate stable transmission.

†Total for the variable categories may not add to $N$ in each column due to missing values.

$\ddagger$ Non-malaria febrile illness defined as history of fever or temperature $\geq 37.5^{\circ} \mathrm{C}$ and a negative malaria test. 

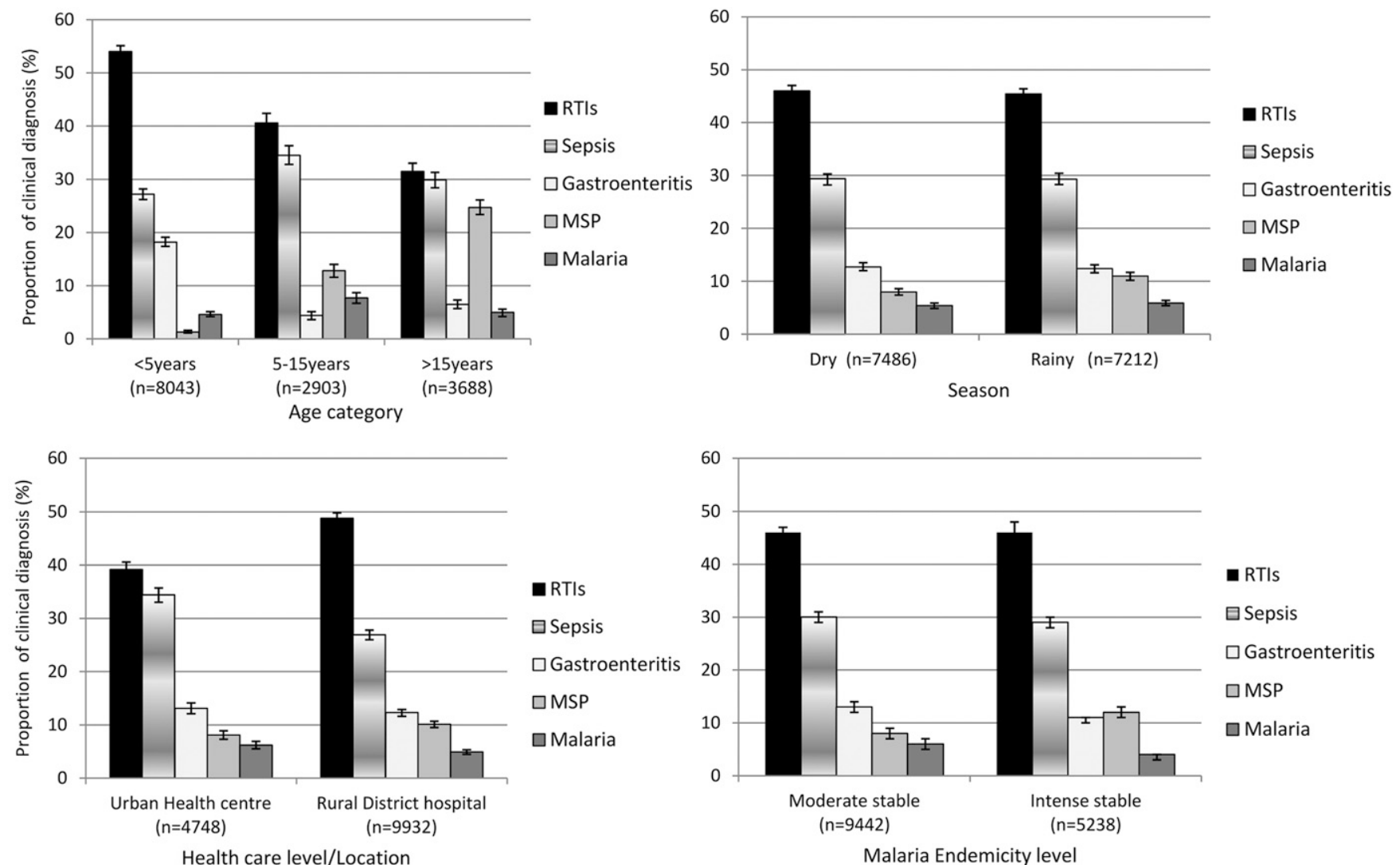

$\mathrm{RTI}=$ Respiratory tract infection; MSP = Musculoskeletal pain

${ }^{*} \mathrm{NMFI}$ is defined as as history of fever or temperature $\geq 37.5^{\circ} \mathrm{C}$ and negative malaria test result

†Malaria endemicity: Intense stable transmission=Chikhwawa; Moderate stable transmission= Ndirande and Thyolo

FIGURE 1. Common clinical diagnoses among outpatients with non-malaria febrile illness (NFMI) in Malawi.*

and lower in rural Chikhwawa district hospital (OR $=0.74 ; 95 \%$ $\mathrm{Cl}=0.69-0.78$ ). In analysis including all possible diagnoses a patient received simultaneously, URTI, GE, LRTI, and sepsis diagnoses were associated with increased odds of antibiotic prescription. Season was not a significant predictor of prescribing antibiotics for NMFI.

\section{DISCUSSION}

This large facility-based malaria surveillance study conducted in different malaria transmission settings in southern Malawi demonstrated that the majority of outpatients with fevers were due to NMFI. The proportion of outpatients with NMFI was lowest in school age children compared to both young children and adults in all settings. Respiratory tract infections and sepsis were the most common clinical diagnoses among patients with NMFls in all age groups and settings. Musculoskeletal pain was frequently diagnosed in both school age children and adults while GE was frequently diagnosed in young children compared to school age children and adults. The distribution pattern of clinical diagnoses did not vary by season in this study. Antibiotics were frequently prescribed in all age groups and settings. Two antibiotics, cotrimoxazole and amoxicillin, were prescribed for all common conditions and health workers prescribed antibiotics for conditions such as musculoskeletal pain and malaria that are not considered bacterial in origin.
Our assessment of the burden of NMFI according to season, urban versus rural location, malaria endemicity, level of health care, and age is more comprehensive than that in previous studies. ${ }^{14,22}$ This study found that the burden of NMFIs was higher in young children and adults than schoolaged children in all settings in the study. Sub-Saharan Africa including Malawi has the highest mortality rate of children younger than 5 years in the world, and more than $50 \%$ of the deaths are due to infectious diseases that are preventable and/or treatable. ${ }^{23}$ However, community-based treatment algorithms for young children with fever are often limited to the diagnosis and treatment of malaria. Furthermore, local investigations to identify and understand common and treatable etiological causes of NMFls may improve management of NMFIs and contribute to reducing the high morbidity and mortality in children younger than 5 years in these settings.

Respiratory tract infections, especially URTIs, were the most common clinical diagnosis in all age-groups and settings. Studies in children in sub-Saharan Africa have similarly found RTls to be the most common cause of acute febrile illness. ${ }^{10,12-14,24}$ However, our results also suggest that some of the clinical diagnoses made by the health workers in patients with NMFls were likely inaccurate. Sepsis was diagnosed in almost one-third of cases; however, etiological studies in adults or children in similar settings have shown that only a very small proportion of outpatient fevers, ranging from $1.3 \%$ to $4.2 \%$, are caused by bloodstream infections. ${ }^{9,12,25} \mathrm{~A}$ 


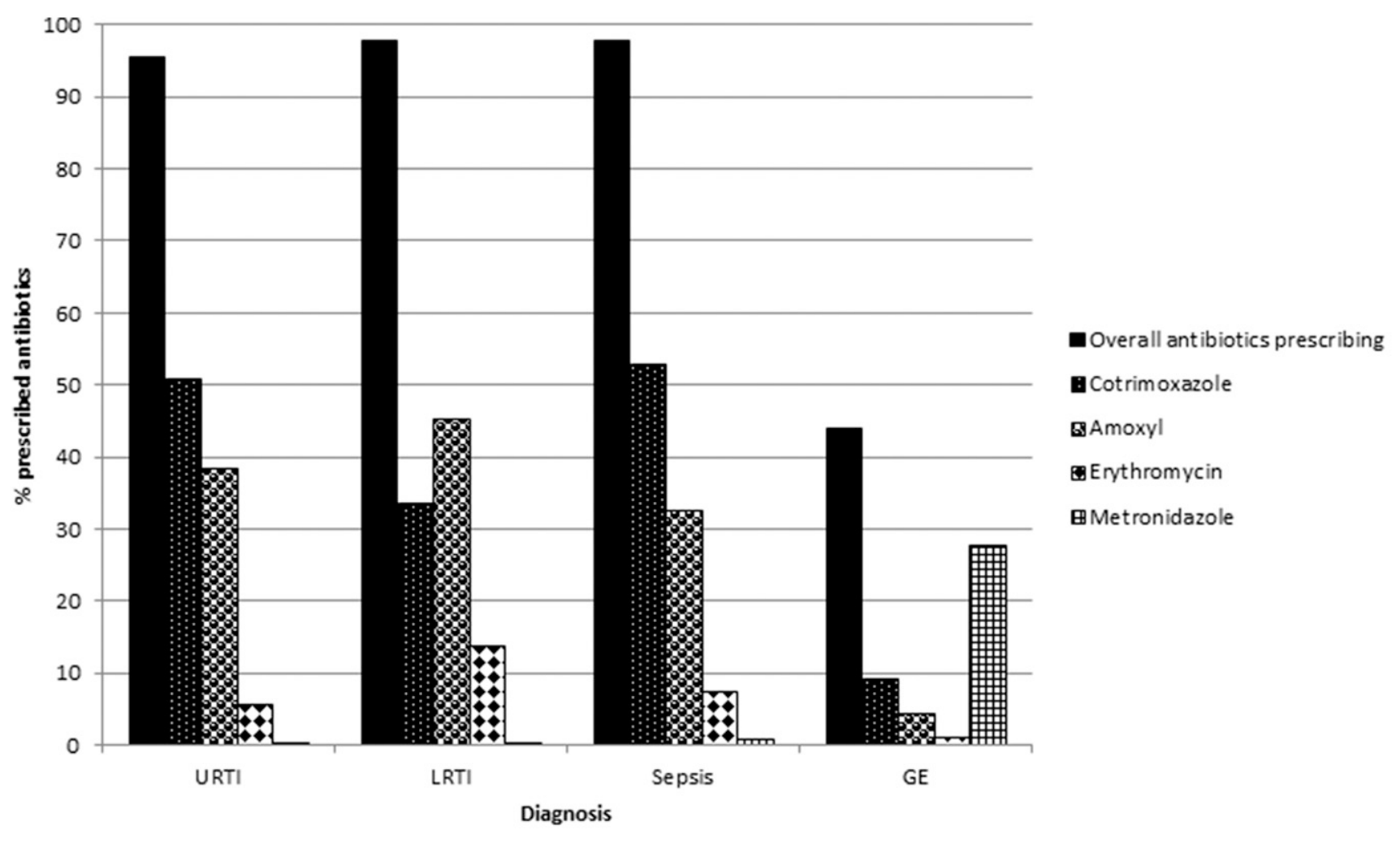

URTI=Upper respiratory tract infection, LRTI = Lower respiratory tract infection, GE = Gastroenteritis

${ }^{*} \mathrm{NMFI}$ is defined as history of fever or temperature $237.5^{\circ} \mathrm{C}$ and a negative malariatest

FIGURE 2. Common antibiotics prescribed among outpatients with non-malaria febrile illness (NMFI) by clinical diagnosis in southern Malawi. ${ }^{*}$

review of studies on bloodstream infections in hospitalized patients who had blood culture taken has reported proportions of only $13.5 \%$ in adults and $8.2 \%$ in children, ${ }^{26}$ further confirming that the observed clinical diagnoses of sepsis even among the sickest patients are overestimates.

For all clinical diagnoses in our study, healthcare workers predominantly prescribed two antibiotics, cotrimoxazole and amoxicillin, and they were often prescribed for diagnoses that are not associated with bacterial infection. These two antibiotics were most frequently available in facility pharmacies. Health workers in most poor resource settings have frequently cited limited resources, drug stock-outs, and lack of diagnostic tests such as blood cultures as a hindrance to optimal patient management. ${ }^{4,6}$ In addition, lack of health workers' knowledge and skills as well as appropriate management algorithms for patients with NMFls could partly explain why patients were frequently prescribed antibiotics for clinical diagnoses that most likely did not require antibiotics such as musculoskeletal pain, URTIs, and GE. Evidence suggests that most URTIs and GE do not require antibiotic therapy. ${ }^{27-29}$ This phenomenon has been observed in other studies, as well. ${ }^{4,14,30-32}$ With the increased use of mRDTs to confirm malaria diagnosis, overprescription of antimalarial drugs is being replaced by overuse of antibacterial agents. ${ }^{30,33}$

In addition to the concern that healthcare providers prescribe antibacterial too frequently, our results also raise the possibility that the available antibiotics will not cure bacterial infections. Antifolate resistance is widespread in Malawi. ${ }^{34}$ Thus, even if some of the clinical diagnoses were accurate, the common prescription of trimethoprim-sulfamethoxazole would not be effective for invasive bacterial infection. Our results highlight the need of providing not only knowledge and skills to health workers to reduce overprescription of antibiotics but also all the required resources including medications and diagnostic tools to effectively manage patients with NMFIs.

The major strength of our study is that our large facilitybased surveillance study included all age-groups in the universal malaria testing era and has reported on current healthcare workers' management practices and prevalence of NMFls in different seasons and settings. ${ }^{35}$ The lack of standardization of clinical diagnoses made by health workers limited our ability to conclude definitive diagnoses for NMFls and could not assess whether antibiotics were appropriate in any given case. Finally, the presence of the study team at the facilities could have led to changes in clinical practice at the facility. However, because our study team was only abstracting data at the patient exit points, we do not believe our presence had a significant impact on diagnosis and treatment practices. Our results suggest that health workers may provide inaccurate clinical diagnoses and inconsistent medication prescriptions in patients with NMFls. Misuse of antibiotics leading to promotion of antimicrobial resistance is a concern of global importance in need of urgent attention, and this includes improving management of NMFls. ${ }^{3}$ Implementations of interventions and programs such as point-of care diagnostics, management algorithms, and training of healthcare workers could assist peripheral health workers make more accurate diagnoses in NMFI patients and provide 
TABLE 3

Factors associated with antibiotic prescription among outpatients with non-malaria febrile illness in Malawi*

\begin{tabular}{|c|c|c|c|c|}
\hline \multirow[b]{2}{*}{ Characteristic } & \multicolumn{2}{|c|}{ Bivariate } & \multicolumn{2}{|c|}{ Multivariable } \\
\hline & OR $(95 \% \mathrm{Cl})$ & $P$-value & OR $(95 \% \mathrm{Cl}) \dagger$ & $P$-value \\
\hline \multicolumn{5}{|l|}{ Malaria endemicity/siteł } \\
\hline Ndirande & - & & - & \\
\hline Thyolo & $1.25(1.14-1.36)$ & $\leq 0.0001$ & $1.21(1.13-1.29)$ & $\leq 0.0001$ \\
\hline Chikhwawa & $1.04(0.95-1.13)$ & & $0.74(0.69-0.78)$ & \\
\hline \multicolumn{5}{|l|}{ Level of health care§ } \\
\hline Health center & - & & & \\
\hline District hospital & $1.14(1.06-1.23)$ & 0.0007 & - & - \\
\hline \multicolumn{5}{|l|}{ Age (years) } \\
\hline$<5$ & $2.21(2.04-2.37)$ & $\leq 0.0001$ & $1.37(1.29-1.45)$ & $\leq 0.0001$ \\
\hline $5-15$ & $1.69(1.53-1.88)$ & & $1.07(0.99-1.15)$ & \\
\hline$>15$ & - & - & - & - \\
\hline \multicolumn{5}{|l|}{ Axillary temperature } \\
\hline 37.5 to $<39.5$ & - & - & & \\
\hline$>39.5$ & $0.99(0.80-1.22)$ & 0.9 & & \\
\hline \multicolumn{5}{|l|}{ Clinician diagnosis (yes vs. no) } \\
\hline Upper respiratory tract infection & $8.5(7.2-10.0)$ & $\leq 0.0001$ & $37.6(33.6-42.0)$ & $\leq 0.0001$ \\
\hline Gastroenteritis & $0.40(0.36-0.4)$ & $\leq 0.0001$ & $1.68(1.56-1.83)$ & $\leq 0.0001$ \\
\hline Musculoskeletal pain & $0.06(0.06-0.07)$ & $\leq 0.0001$ & $0.22(0.20-0.24)$ & $\leq 0.0001$ \\
\hline Malaria & $0.15(0.13-0.17)$ & $\leq 0.0001$ & $0.11(0.10-0.12)$ & $\leq 0.0001$ \\
\hline Lower respiratory tract infection & $14.0(8.8-22.4)$ & $\leq 0.0001$ & $91.5(65.7-127.4)$ & $\leq 0.0001$ \\
\hline Sepsis & $16.4(13.5-19.9)$ & $\leq 0.0001$ & $4.1(55.4-74.2)$ & $\leq 0.0001$ \\
\hline All other diagnoses & $1.97(1.78-2.19)$ & $\leq 0.0001$ & $4.6(4.3-4.9)$ & $\leq 0.0001$ \\
\hline \multicolumn{5}{|l|}{ Dry (vs. rainy) season } \\
\hline Dry & $1.07(1.00-1.15)$ & 0.05 & $0.97(0.92-1.02)$ & 0.2 \\
\hline
\end{tabular}

${ }^{*}$ Defined as a history of fever or temperature $\geq 37.5^{\circ} \mathrm{C}$ and a negative malaria.

$\dagger$ Final model including geographical location, age, clinician diagnosis, and season variables with a $P$-value $<0.05$ or caused substantial change in the coefficients of other variables in the model. Wald $P$-values and confidence limits are shown for each variable included in the model.

$\ddagger$ Malaria endemicity: Chikhwawa = rural lowland with intense stable transmission; Thyolo = rural highland with moderate stable transmission; Ndirande = urban highland with moderate stable transmission.

$\S$ Not included in the initial multivariable model as it is collinear with the malaria endemicity variable. This variable was highly correlated with the endemicity/study site variable. When both were added, none was significant. Endemicity variable was left as it added more information to the analysis than "level of health care."

effective and cost-efficient patient care in resource-limited settings. ${ }^{4,36-38}$ Point-of-care rapid diagnostic tests such as for influenza infection or use of biological markers to identify patients with bloodstream infections could lead to more improved diagnosis and target treatment of NMFIs. ${ }^{38,39}$ Future studies to evaluate diagnostic testing and treatment algorithms should assess their impact on patient care, cost, and the spread of antimicrobial resistance.

Received October 4, 2018. Accepted for publication April 6, 2020.

Published online June 22, 2020.

Acknowledgments: We thank the patients who participated in this study, and we are grateful for the hard work and inputs from the Malawi ICEMR field team, Malawi National Malaria Control program, district health teams, and hospital staff of Ndirande in Blantyre, Chikhwawa, and Thyolo, and the management of Queen Elizabeth Central hospital. We thank Malaria Alert Center staff for administration and logistical support.

Financial support: This work was supported by a grant from the U.S. National Institute of Allergy and Infectious Diseases, Bethesda, MD (U19AI089683 to T. E. T.), for the Malawi International Center for Excellence in Malaria Research and K24Al114996 to M. K. L.

Authors' addresses: Atupele Kapito-Tembo, Malaria Alert Center, University of Malawi College of Medicine, Blantyre, Malawi, and Boston University School of Public Health, Boston, MA, E-mail: atupelekapito@gmail.com. Don Mathanga and Andrew Bauleni, Malaria Alert Center, University of Malawi College of Medicine, Blantyre, Malawi, E-mails: dmathang@mac.medcol.mw and abauleni@ mac.medcol.mw. Osward Nyirenda and Paul Pensulo, Blantyre Malaria Project, University of Malawi College of Medicine, Blantyre, Malawi, E-mails: omnyirenda@gmail.com and paul.pensulo@ gmail.com. Doreen Ali, Malawi National Malaria Control Program,
Lilongwe, Malawi, E-mail: alidoreen@yahoo.com. Clarissa Valim, Department of Immunology and Infectious Diseases, Harvard School of Public Health, Boston, MA, and Boston University School of Public Health, Boston, MA, E-mail: clarissa.valim@gmail.com. Terrie E. Taylor, Blantyre Malaria Project, University of Malawi College of Medicine, Blantyre, Malawi and College of Osteopathic Medicine, Michigan State University, East Lansing, MI, E-mail: ttmalawi@ msu.edu. Miriam K. Laufer, Center for Vaccine Development and Global Health, University of Maryland School of Medicine, Baltimore, MD, E-mail: mlaufer@som.umaryland.edu.

\section{REFERENCES}

1. World Health Organization, 2015. Guidelines for the Treatment of Malaria, 3rd edition. Geneva, Switzerland: World Health Organization.

2. D'Acremont V, Lengeler C, Genton B, 2010. Reduction in the proportion of fevers associated with Plasmodium falciparum parasitaemia in Africa: a systematic review. Malar J 9: 240.

3. World Health Organization, 2013. WHO Informal Consultation on Fever Management in Peripheral Health Care Settings: A Global Review of Evidence and Practice. Geneva, Switzerland: World Health Organization.

4. Hooft AM, Ripp K, Ndenga B, Mutuku F, Vu D, Baltzell K, Masese LN, Vulule J, Mukoko D, LaBeaud AD, 2017. Principles, practices and knowledge of clinicians when assessing febrile children: a qualitative study in Kenya. Malar J 16: 381.

5. Chipwaza B, Mugasa JP, Mayumana I, Amuri M, Makungu C, Gwakisa PS, 2014. Community knowledge and attitudes and health workers' practices regarding non-malaria febrile illnesses in Eastern Tanzania. PLoS Negl Trop Dis 8: e2896.

6. Baltzell K, Elfving K, Shakely D, Ali AS, Msellem M, Gulati S, Martensson A, 2013. Febrile illness management in children under five years of age: a qualitative pilot study on primary health care workers' practices in Zanzibar. Malar J 12: 37. 
7. Johansson EW, Kitutu FE, Mayora C, Awor P, Peterson SS, Wamani H, Hildenwall H, 2016. It could be viral but you don't know, you have not diagnosed it: health worker challenges in managing non-malaria paediatric fevers in the low transmission area of Mbarara district, Uganda. Malar J 15: 197.

8. Lubinski C, 2016. ASTMH 2016: Initiative Engages Low-Income Countries in Responses to Antimicrobial Resistance Science Speaks: Global ID News. http://sciencespeaksblog.org/2016/ 11/17/astmh-2016-initiative-engages-low-income-countriesin-responses-to-antimicrobial-resistance/. Accessed March 19, 2017.

9. Hildenwall H, Amos B, Mtove G, Muro F, Cederlund K, Reyburn H, 2016. Causes of non-malarial febrile illness in outpatients in Tanzania. Trop Med Int Health 21: 149-156.

10. Elfving $\mathrm{K}$ et al., 2016. Acute uncomplicated febrile illness in children aged 2-59 months in Zanzibar-aetiologies, antibiotic treatment and outcome. PLoS One 11: e0146054.

11. Prasad N, Murdoch DR, Reyburn H, Crump JA, 2015. Etiology of severe febrile illness in low- and middle-income countries: a systematic review. PLoS One 10: e0127962.

12. D'Acremont V, Kilowoko M, Kyungu E, Philipina S, Sangu W, Kahama-Maro J, Lengeler C, Cherpillod P, Kaiser L, Genton B, 2014. Beyond malaria--causes of fever in outpatient Tanzanian children. N Engl J Med 370: 809-817.

13. Mahende $C$, Ngasala B, Lusingu J, Butichi A, Lushino $P$, Lemnge $\mathrm{M}$, Premii Z, 2014. Aetiology of acute febrile episodes in children attending Korogwe district hospital in North-Eastern Tanzania. PLoS One 9: e104197.

14. Ndhlovu M, Nkhama E, Miller JM, Hamer DH, 2015. Antibiotic prescribing practices for patients with fever in the transition from presumptive treatment of malaria to 'confirm and treat' in Zambia: a cross-sectional study. Trop Med Int Health 20: 1696-1706.

15. Gething PW, Kirui VC, Alegana VA, Okiro EA, Noor AM, Snow RW, 2010. Estimating the number of paediatric fevers associated with malaria infection presenting to Africa's public health sector in 2007. PLoS Med 7: e1000301.

16. Herlihy JM, D'Acremont V, Burgess DCH, Hamer DH, 2016. Diagnosis and treatment of the febrile child. Disease Control Priorities (Volume 2): Reproductive, Maternal, Newborn, and Child Health, 3rd Edition. Washington, DC: World Bank.

17. Kiemde F, Spijker R, Mens PF, Tinto H, Boele M, Schallig HD, 2016. Aetiologies of non-malaria febrile episodes in children under 5 years in sub-Saharan Africa. Trop Med Int Heal 21: 943-955.

18. Crump JA, Gove S, Parry CM, 2011. Management of adolescents and adults with febrile illness in resource limited areas. BMJ 343: d4847.

19. Ministry of Natural Resources E and E. Malawi Meteorological Services: Climate of Malawi, 2014. Available at: http:// www.metmalawi.com/climate/climate.php. Accessed August 11, 2014.

20. Bennett A, Kazembe L, Mathanga DP, Kinyoki D, Ali D, Snow RW, Noor AM, 2013. Mapping malaria transmission intensity in Malawi, 2000-2010. Am J Trop Med Hyg 89: 840-849.

21. Blantyre District Council, 2007. Blantyre Socio-Economic Profile 2007-2010. Available at: https://www.yumpu.com/en/document/ view/34833884/blantyre-socio-economic-profile-2007-2010draftscotland-malawi-. Accessed October 4, 2019.

22. Saweri OP, Hetzel MW, Mueller I, Siba PM, Pulford J, 2017. The treatment of non-malarial febrile illness in Papua New Guinea: findings from cross sectional and longitudinal studies of health worker practice. BMC Health Serv Res 17: 10.

23. United Nations Inter-agency Group for Child Mortality Estimation (UN IGME), 2019. Levels and Trends in Child Mortality: Report
2019, Estimates developed by the United Nations Inter-agency Group for Child Mortality Estimation. New York, NY: United Nations Children's Fund.

24. Muroa F, Reyburn R, Reyburn H, 2015. Acute respiratory infection and bacteraemia as causes of non-malarial febrile illness in African children: a narrative review. Pneumonia (Nathan) 6: 6-17.

25. Mahende C, Ngasala B, Lusingu J, Butichi A, Lushino P, Lemnge M, Mmbando B, Premji Z, 2015. Bloodstream bacterial infection among outpatient children with acute febrile illness in NorthEastern Tanzania. BMC Res Notes 8: 289.

26. Reddy EA, Shaw AV, Crump JA, 2010. Community-acquired bloodstream infections in Africa: a systematic review and metaanalysis. Lancet Infect Dis 10: 417-432.

27. Kotloff KL et al., 2013. Burden and aetiology of diarrhoeal disease in infants and young children in developing countries (the global enteric multicenter study, GEMS): a prospective, case-control study. Lancet 382: 209-222.

28. Gutierrez MF, Matiz A, Trespalacios AA, Parra M, Riano M, Mercado M, 2006. Virus diversity of acute diarrhea in tropical highlands. Rev Latinoam Microbiol 48: 17-23.

29. Kutty N, 2011. Treating children without antibiotics in primary healthcare. Oman Med J 26: 303-305.

30. Hopkins $\mathrm{H}$ et al., 2017. Impact of introduction of rapid diagnostic tests for malaria on antibiotic prescribing: analysis of observational and randomised studies in public and private healthcare settings. BMJ 356: j1054.

31. D'Acremont V, Kahama-Maro J, Swai N, Mtasiwa D, Genton B, Lengeler C, 2011. Reduction of anti-malarial consumption after rapid diagnostic tests implementation in Dar es Salaam: a before-after and cluster randomized controlled study. Malar $J$ 10: 107.

32. Filice GA, Drekonja DM, Thurn JR, Hamann GM, Masoud BT, Johnson JR, 2015. Diagnostic errors that lead to inappropriate antimicrobial use. Infect Control Hosp Epidemiol 36: 949-956.

33. Bruxvoort KJ et al., 2017. The impact of introducing malaria rapid diagnostic tests on fever case management: a synthesis of ten studies from the ACT consortium. Am J Trop Med Hyg 97: 1170-1179.

34. Makoka MH, Miller WC, Hoffman IF, Cholera R, Gilligan PH, Kamwendo D, Malunga G, Joaki G, Martinson F, Hosseinipour MC, 2012. Bacterial infections in Lilongwe, Malawi: aetiology and antibiotic resistance. BMC Infect Dis 12: 67.

35. Johansson EW, Selling KE, Nsona H, Mappin B, Gething PW, Petzold M, Peterson SS, Hildenwall H, 2016. Integrated paediatric fever management and antibiotic over-treatment in Malawi health facilities: data mining a national facility census. Malar J 15: 396.

36. Ngasala B, Mubi M, Warsame M, Petzold MG, Massele AY, Gustafsson LL, Tomson G, Premji Z, Bjorkman A, 2008. Impact of training in clinical and microscopy diagnosis of childhood malaria on antimalarial drug prescription and health outcome at primary health care level in Tanzania: a randomized controlled trial. Malar J 7: 199.

37. Chappuis F, Alirol E, d'Acremont V, Bottieau E, Yansouni CP, 2013. Rapid diagnostic tests for non-malarial febrile illness in the tropics. Clin Microbiol Infect 19: 422-431.

38. Centers for Disease Control and Prevention, 2013. Guidance for Clinicians on the Use of Rapid Influenza Diagnostic Tests. Available at: http://www.cdc.gov/flu/professionals/diagnosis/ clinician_guidance_ridt.htm.

39. Decuypere S et al., 2016. Towards improving point-of-care diagnosis of non-malaria febrile illness: a metabolomics approach. PLoS Negl Trop Dis 10: e0004480. 